\title{
Acetylcholine Promotes ROS Detoxification Against Hypoxia/reoxygenation-Induced Oxidative Stress Through FoxO3a/PGC-1 $\alpha$ Dependent Superoxide Dismutase
}

\author{
Lei Sun Wei-Jin Zang Hao Wang Mei Zhao Xiao-Jiang Yu Xi He Yi Miao \\ Jun Zhou
}

Department of Pharmacology, Xi'an Jiaotong University Health Science Center, Xi'an, Shaanxi, P.R. China

\section{Key Words}

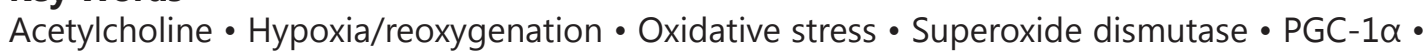
FoxO3a

\begin{abstract}
Background/Aims: Acetylcholine (ACh) is known to modulate the cardiac redox environment and thereby suppress reactive oxygen species (ROS) generation during oxidative stress. However, there is little information about its regulation on ROS clearance. Here we investigate the beneficial effects of ACh on superoxide dismutase (SOD) as key ROS-detoxifying enzyme system in cultured rat cardiomyoblasts. Methods: H9c2 cells were subjected to hypoxia/ reoxygenation $(H / R)$ to mimic oxidative stress. Western blot was used to detect the expression of SOD and related signaling molecules. Specific protein knockdown was performed with siRNA transfection. Results: ACh treatment on the beginning of reoxygenation decreased ROS and apoptosis. ACh increased ATP synthesis and mitochondrial DNA. Furthermore, ACh significantly reversed $\mathrm{H} / \mathrm{R}$-induced reduction in protein expressions and activities of SOD. ACh stimulated peroxisome proliferator activated receptor $\gamma$ co-activator $1 \alpha$ (PGC-1 $\alpha$ ) and decreased forkhead box subfamily O3a (FoxO3a) phosphorylation. Atropine (muscarinic receptor antagonist) abolished the cytoprotection afforded by ACh. PGC-1 $\alpha$ siRNA blocked ACh-induced invigorating effects on SOD2, whereas it did not alter SOD1 and FoxO3a phosphorylation. FoxO3a siRNA drastically decreased the expressions of SOD2 and PGC-1 $\alpha$, while it did not affect SOD1. Conclusion: ACh activates SOD2 within mitochondria through FoxO3a/PGC-1 $\alpha$ pathway and up-regulates SOD1 in the cytoplasm, thus protecting against oxidative injury induced by $\mathrm{H} / \mathrm{R}$. Our findings provide new insights into mechanisms underlying the cardioprotection of ACh on ROS detoxifying.
\end{abstract}




\section{Cellular Physiology and Biochemistry}

Cell Physiol Biochem 2014;34:1614-1625

\begin{tabular}{l|l}
\hline DOI: $10.1159 / 000366364$ & C 2014 S. Karger AG, Basel
\end{tabular}

Sun et al: ACh Increases Superoxide Dismutase

\section{Introduction}

Ischemic heart disease or myocardial ischemia/reperfusion (I/R) is a major cause of morbidity and mortality worldwide, which is associated with increased formation of reactive oxygen species (ROS) [1-3]. Excessive ROS produced by the nicotinamide dinucleotide phosphate (NADPH) oxidase, mitochondria, or the xanthine oxidase (XO) leads to DNA, protein and lipid modifications, as well as activates oxidative stress-related signaling pathways which cause further damage [4]. Because of the deleterious effects of ROS, the cell has evolved complex enzyme systems to detoxify ROS throughout the cell. Superoxide dismutase (SOD) is the first line of antioxidant defense against ROS, and SOD is the only enzyme that can detoxify superoxide. SOD acts by converting superoxide to hydrogen peroxide, which can subsequently be converted to water by catalase or peroxiredoxin [5]. There are two major intracellular SOD isoforms. The copper-zinc SOD isoform (SOD1) is present in the cytosol and the manganese SOD isoform (SOD2) is primarily located in mitochondria [6]. In the myocardial I/R, an abrupt burst of ROS, particularly mitochondrial ROS production, leads to SOD consumption; on the other hand, SOD synthesis decreased due to the energy deficiency. Cumulatively these changes result in excessive ROS accumulation in myocardium, reduced anti-oxidative activity and mitochondrial dysfunction [7]. The increased mitochondrial ROS production can lead to opening of the mitochondrial permeability transition pore, thereby instigating a chain of events that leads to both apoptotic and necrotic cardiomyocyte death $[8,9]$. Accordingly, improving oxidative defense systems to counter the imbalance of ROS remains an attractive strategy for prevention and therapy of I/R injury.

Recently several transcription factors have been recognized in the regulation of antioxidative enzyme. Peroxisome proliferator activated receptor $\gamma$ co-activator $1 \alpha$ (PGC-1 $\alpha$ ) plays a pivot role in the modulation of mitochondrial biogenesis and oxidative metabolism, which promotes the transcription of several key ROS-detoxifying genes [10-12]. Another critical mediator of ROS homeostasis is the forkhead box subfamily 0 (FoxO). The function of FoxO factors, in particular Fox03a, in cardiac pathophysiology is an active area of research. FoxO proteins promote oxidative stress resistance by binding to the promoters of the genes encoding SOD2, catalase and peroxiredoxin 3 [13-15]. Intriguingly, it is newly reported that Fox03a and PGC-1 $\alpha$ interact directly in the vascular endothelial cells and are recruited to the same promoter regions to regulate mitochondrial oxidative stress protection gene SOD2 [16]. Thus, transcription factors are emerging as master signaling integrators, which translate various stimuli into dynamic gene expression programs that influence potent functions of related proteins; dysregulation of these proteins may ultimately lead to disease.

Imbalances in the autonomic nervous system have been implicated in the pathogenesis and progression of cardiovascular diseases. Increased vagal activity reduces cardiovascular risk factors and improves prognosis [17-19]. Recently, vagal nerve stimulation (VNS) has been used as a promising therapeutic approach for chronic heart failure [20-22]. Strikingly, Tsutsumi el al. has demonstrated that VNS suppresses ROS overproduction and resets the myocardial redox imbalance [23]. Consistently, our previous studies have shown that $\mathrm{ACh}$, the principle neurotransmitter of vagal nerve, reduces angiotensin $\Pi$ and hypoxiainduced ROS in H9c2 cells [24, 25]. Recent study in our laboratory has indicated that ACh inhibits mitochondria-, xanthine oxidase- and NAPDH oxidase-derived ROS formation thus protecting cardiomyocytes against hypoxia/reoxygenation (H/R)-induced oxidative stress [26]. However, at present, there is no evidence to implicate the ROS detoxification mechanism of action for the benefits derived from vagal activation. Therefore, this study has been undertaken in order to determine the role of antioxidant enzyme SOD in the protective effects of ACh against H/R-induced oxidative stress in H9c2 cells. In addition, we determined Fox03a/PGC-1 $\alpha$ signaling pathway as SOD-associated regulatory mechanism. 


\section{Cellular Physiology and Biochemistry}

Cell Physiol Biochem 2014;34:1614-1625

DOI: $10.1159 / 000366364$

Published onIIne: November 03, 2014

2014 S. Karger AG, Basel

www.karger.com/cpb

Sun et al.: ACh Increases Superoxide Dismutase

\section{Materials and Methods}

Cell culture, hypoxia/reoxygenation induction and ACh treatment

H9c2 cells, derived from rat embryonic ventricular cardiomyocytes, were purchased from American Type Culture Collection (ATCC, Manassas, VA, USA). H9c2 cell line has been confirmed as a valid in vitro tool for the examination of cardiovascular pathophysiology [27]. Cells were maintained in DMEM supplemented with 10\% FBS (v/v) and 1\% penicillin/streptomycin (Sigma, St Louis, MO, USA) (v/v) in a humidified incubator with $95 \%$ air and $5 \% \mathrm{CO}_{2}$ at $37^{\circ} \mathrm{C}$. After grown to $80 \%$ confluence, cells were starved in serumfree DMEM for $12 \mathrm{hrs}$, then subjected to modified ischemia-mimetic solution (135 $\mathrm{mM} \mathrm{NaCl}, 8 \mathrm{mM} \mathrm{KCl}$, $0.33 \mathrm{mM} \mathrm{NaH}_{2} \mathrm{PO}_{4}, 0.5 \mathrm{mM} \mathrm{MgCl}_{2}, 5.0 \mathrm{mM}$ Hepes, $1.8 \mathrm{mM} \mathrm{CaCl}_{2}$, and $20 \mathrm{mM}$ lactate, $\mathrm{pH} 6.80$ ) and hypoxia for $8 \mathrm{hrs}$ in a modulator incubator gassed with $1 \% \mathrm{O}_{2}, 5 \% \mathrm{CO}_{2}$, and $94 \% \mathrm{~N}_{2}$ ). For reoxygenation, cells were incubated in serum-free DMEM at $37^{\circ} \mathrm{C}$ in a humidified atmosphere of $5 \% \mathrm{CO}_{2}$ for $1 \mathrm{hr}, 2 \mathrm{hrs}, 4 \mathrm{hrs}, 8 \mathrm{hrs}$, $16 \mathrm{hrs}$ and $24 \mathrm{hrs}$ respectively. To investigate the effect of ACh on ROS generation, cells were treated with various concentrations of ACh $\left(10^{-6}\right.$ to $\left.10^{-3} \mathrm{M}\right)$ at the onset of reoxygenation. ACh $\left(10^{-5} \mathrm{M}\right)$ was also used in combination with atropine $\left(10^{-4} \mathrm{M}\right.$, a non-selective muscarinic receptor antagonist) as decribed in our previous study.

\section{Measurement of intracellular ROS}

Generation of ROS was monitored by the measurement of superoxide generation using the fluorescent probes dihydroethidium (DHE, Beyotime, Haimen, China). Briefly, H9c2 cells in 24-well plates $\left(3 \times 10^{4}\right.$ per well) were incubated with DHE $(8 \mu \mathrm{M})$ for $30 \mathrm{~min}$ at $37^{\circ} \mathrm{C}$ in the dark, followed by washing with phosphate buffer solution. The fluorescence was measured with an inverted fluorescence microscope (TE-2000U, Nikon, Japan) and analyzed using Image Pro-Plus 6.0 software (Media Cybernetics, Silver Spring, MD, USA). The values were expressed as percent of integrated optical density (IOD) in the control.

Western blot

Cells were harvested and centrifuged at 1,000 r.p.m. for 5 min after lysing with RIPA buffer (Beyotime) containing $1 \mathrm{mM}$ phenylmethanesulfonyl fluoride (PMSF, Beyotime Haimen, China) for $10 \mathrm{~min}$ at $4^{\circ} \mathrm{C}$. The lysates were then centrifuged at 11,000 r.p.m. for $15 \mathrm{~min}$ at $4^{\circ} \mathrm{C}$ and then boiled for $5 \mathrm{~min}$ in loading buffer. Protein concentration was determined using the Bicinchoninic acid protein assay kit (Beyotime, Haimen, China). Samples (30 $\mu$ g per lane) were loaded on $12 \%$ sodium dodecyl sulfate-polyacrylamide gel electrophoresis (SDS-PAGE). The separated proteins were transferred to polyvinylidene difluoride membranes followed by blocking of the membrane with $5 \%$ nonfat milk (w/v) in TBST (10 mM Tris, 150 $\mathrm{mM} \mathrm{NaCl}, 0.1 \%$ Tween 20) for $4 \mathrm{hrs}$ at room temperature. Membranes were probed for the protein levels of SOD1, SOD2 (Abcam Inc., Cambridge, MA, USA), PGC-1 $\alpha$ (Santa Cruz Biotechnology, Delaware, CA, USA), Fox03a and phosphorylation of Fox03a (Thr32) (Cell Signaling Technology, Beverly, MA, USA) using specific primary antibodies followed by a horseradish peroxidase -conjugated appropriate secondary antibody (Beyotime, Haimen, China). Membranes were probed with GAPDH primary antibody as a protein loading control. After being washed, protein bands were shown using ECL-Plus reagent (Pierce, Rockford, IL, USA) and quantified by scanning densitometry.

\section{Real-time polymerase chain reaction (PCR) for mitochondrial DNA}

Total DNA and mitochondrial DNA (mtDNA) were extracted using a QIAamp DNA Mini kit (Qiagen, Hilden, Germany) and real time PCR was done using 18SrRNA primers (forward: CATTCGAACGTCTGCCCTATC; reverse: CCTGCTGCCTTCCTTGGA) for nuclear target sequence and mitochondrial D-loop primers (forward: TGGTTCATCGTCCATACGTT; reverse: TGACGGCTATGTTGAGGAAG) for mitochondrial target. Primers were synthesized by GenScript Biotechnology Co. Ltd (Nanjing, China). Reactions were performed in a $25 \mu \mathrm{l}$ volume containing $12.5 \mu \mathrm{l} 2 \times \mathrm{SYBR}^{\circledR}$ Premix Ex Taq ${ }^{\mathrm{TM}}$ (TaKaRa Biotechnology, Dalian, China), $1.0 \mu \mathrm{l}$ forward

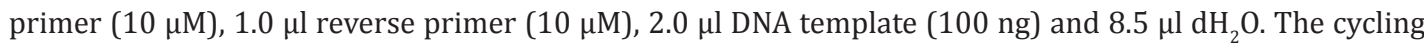
conditions were as follows: $95^{\circ} \mathrm{C}$ for $30 \mathrm{~s}$ followed by 40 cycles of $95^{\circ} \mathrm{C}$ for $5 \mathrm{~s}$ and $60^{\circ} \mathrm{C}$ for $30 \mathrm{~s}$. Each quantitative PCR was performed in triplicate. The ratio of mitochondrial D-loop to $18 \mathrm{~S}$ rRNA was then calculated. Final results are presented as percentage of control. 


\section{Cellular Physiology and Biochemistry}

Cell Physiol Biochem 2014;34:1614-1625

Sun et al.: ACh Increases Superoxide Dismutase

Measurement of intracellular ATP content

Intracellular ATP content was measured using the ATP bioluminescent assay kit (Beyotime, Haimen, China) according to the manufacturer's instructions. Experiments were performed in triplicate.

Measurement of SOD activity

SOD activity was measured using the SOD activity assay kit (Beyotime, Haimen, China) according to the manufacturer's instructions. Experiments were performed in triplicate.

TUNEL

Terminal deoxynucleotidyl transferase-mediated dUTP nick end-labeling (TUNEL) assay was performed using One Step TUNEL Apoptosis Assay Kit (KeyGNE BioTECH, Nanjing, China) according to the manufacturer's protocol. Briefly, cells were grown in 96-well plates. After H/R treatment, cells were fixed with $4 \%$ paraformaldehyde and permeabilized using $0.1 \%$ Triton X-100 in $0.1 \%$ sodium citrate. Then, cells were incubated in TUNEL reaction working solution for $1 \mathrm{hr}$ at $37^{\circ} \mathrm{C}$, and followed by incubation with the fluorescent label DAPI (Beyotime, Haimen, China). The fluorescence was analyzed using an inverted fluorescence microscope (TE-2000U, Nikon, Japan). TUNEL-positive nuclei were expressed as the percentage of apoptotic cells (apoptotic nuclei/total nuclei $\times 100 \%$ ).

SiRNA

The siRNAs oligo (PGC-1 $\alpha$, Fox03a and negative control) were synthesized by Shanghai GenePharma Co. Ltd (Shanghai, China). Transfections were performed using TurboFect siRNA Transfection Reagent (R1401, Thermo Scientific, Waltham, MA, USA) following the manufacturer's instructions with $100 \mathrm{nM}$ of each siRNA. Cells were harvested $48 \mathrm{hrs}$ post-transfection and analyzed by western blot to confirm the knockdown of PGC-1 $\alpha$ or FoxO3a. Parallel cells were subjected to H/R injury and ACh treatment as before.

\section{Statistical analysis}

All experiments were performed at least three times for each determination. Data were expressed as mean \pm SEM. Statistical analysis was performed by Student's $t$-test or one-way analysis of variance (ANOVA) followed by a Tukey's post hoc test for multiple comparisons. A $P$ value $<0.05$ was considered statistically significant. All figures were prepared with Graphpad Prism 5.0 (San Diego, CA, USA).

\section{Results}

ACh reduced ROS level during $H / R$ by MAChR

H9c2 cells were subjected to $8 \mathrm{hrs}$ of hypoxia followed by $1 \mathrm{hr}, 2 \mathrm{hrs}, 4 \mathrm{hrs}, 8 \mathrm{hrs}, 16$ hrs or $24 \mathrm{hrs}$ of oxygenation, respectively. Intracellular superoxide anion production was assessed by DHE fluorescence at different time point. As shown in Fig. $1 \mathrm{~A}$ and B, there was no significant difference in fluorescence intensity between control group (Cells were kept in normal condition) and ischemia group (Cells subjected to hypoxia for $8 \mathrm{hrs}$ without reoxygenation). And the ROS levels elevated to its peak at $2 \mathrm{hrs}$ after reoxygenation and declined slowly thereafter. So $8 \mathrm{hrs}$ of hypoxia followed by $2 \mathrm{hrs}$ reoxygenation was performed to mimic oxidative stress in subsequent experiments. Treatment with ACh at $10^{-6}-10^{-3} \mathrm{M}$ on the beginning of reperfusion attenuated the H/R-induced superoxide in a concentration-dependent way (Fig. 1C, D). More specifically, ACh at $10^{-6} \mathrm{M}$ had no obvious effect on fluorescence intensity compared with $\mathrm{H} / \mathrm{R}$ group. ACh at $10^{-5}-10^{-3} \mathrm{M}$ showed a markedly decreased trend in fluorescence intensity and had no significant difference among different concentrations. Therefore, further studies were performed using lowest effective concentration of ACh at $10^{-5} \mathrm{M}$.

Previous studies have documented that ACh exerts cardioprotective effects against myocardial I/R injury through muscarinic ACh receptor (MAChR). In order to examine whether MAChR plays a key role in ACh-mediated suppression of ROS levels, the cells were administrated with ACh $\left(10^{-5} \mathrm{M}\right)$ and co-treated with atropine (MAChR inhibitor, $10^{-4} \mathrm{M}$ ). DHE fluorescence analysis showed that ACh markedly reduced H/R-induced ROS $(P<$ 


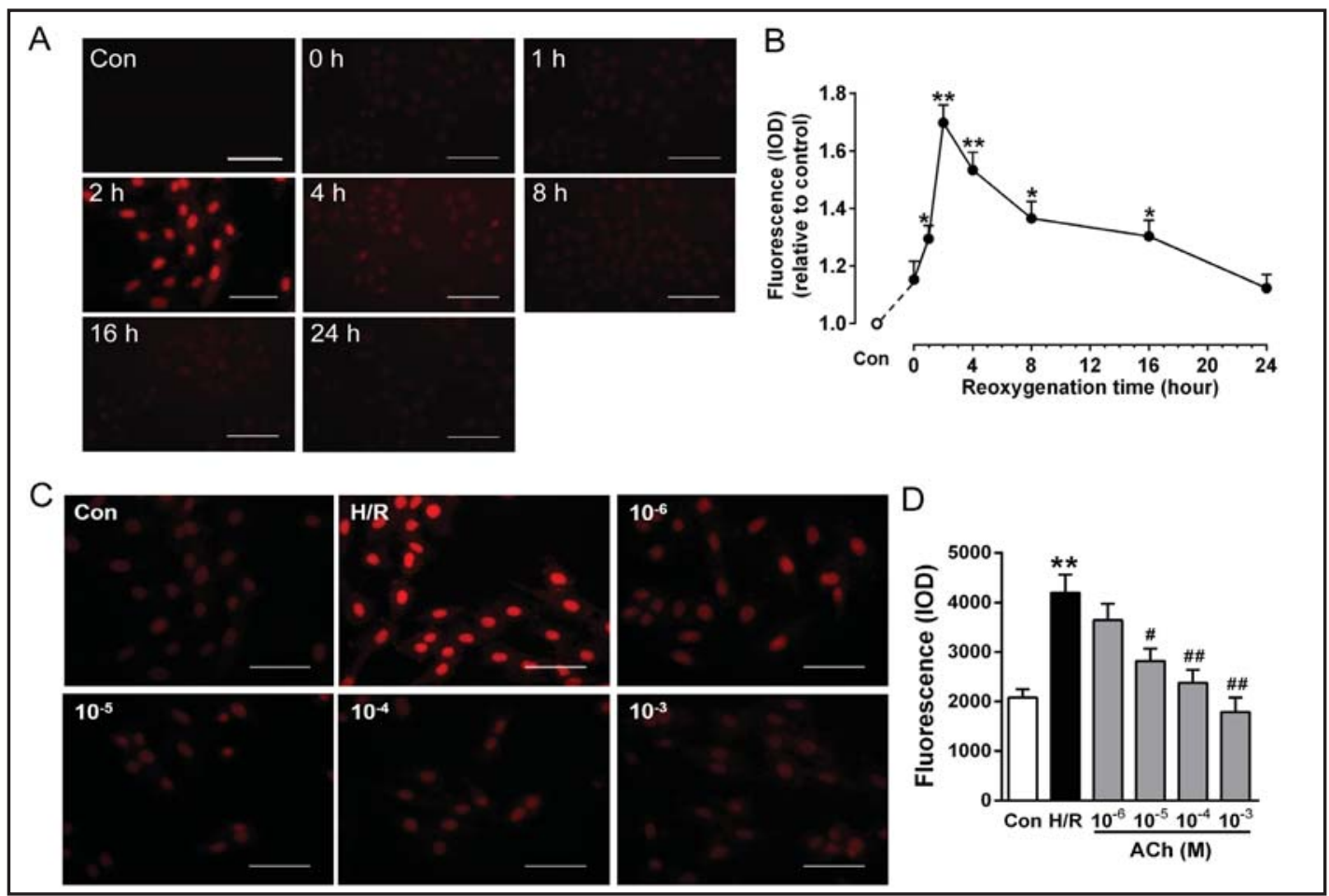

Fig. 1. Change with time of superoxide in response to hypoxia/reoxygenation and ACh reduced superoxide in a concentration dependent way. A-B: Representative fluorescent microscope pictures and IOD line graph of DHE stain in different time point. After 8 hrs hypoxia treatment, ROS fluorescence in H9c2 myocytes was detected at different reoxygenation time separately. ${ }^{*} P<0.05$, ${ }^{* *} P<0.01$ vs. control. C-D: Representative fluorescent microscope pictures and IOD histogram of DHE stain in different concentration of ACh-treated groups. Con: Cells were kept in normal condition; H/R: Cells were treated for $2 \mathrm{hrs}$ of reoxygenation after $8 \mathrm{hrs}$ of hypoxia; $10^{-3}$ to $10^{-6}$ : Cells were treated with $10^{-3}$ to $10^{-6} \mathrm{M}$ ACh during reoxygenation. ${ }^{* *} P<0.01 \mathrm{vs}$. Con; ${ }^{\#} P<0.05,{ }^{\# \# P<0.01 ~ v s . ~ H / R . ~ S c a l e ~ b a r ~ r e p r e s e n t s ~} 200 \mu \mathrm{m}$.

Fig. 2. ACh reduced ROS induced by H/R in H9c2 cells via MAChR. Representative fluorescent microscope pictures and IOD histogram of DHE stain in different groups. Con: Cells were kept in normal condition; Con+ACh: Cells were kept in normal condition and treated with $10^{-5} \mathrm{M} \mathrm{ACh}$ for corresponding time. Con+Atro: Cells were kept in normal condition and added $10^{-4} \mathrm{M}$ atropine for corresponding time. H/R: Cells were subjected to $8 \mathrm{hrs}$ of hypoxia following 2 hrs of reoxygenation; ACh: Cells were treated with $10^{-5} \mathrm{M}$ ACh during reoxygenation; $\mathrm{ACh}+$ Atro: Cells were treated with $10^{-5} \mathrm{M}$ ACh and $10^{-4} \mathrm{M}$ atropine during reoxygenation. ${ }^{* *} P<0.01$ vs. Con; ${ }^{\# \#} P<$ 0.01 vs. H/R; ${ }^{\S} P<0.05$ vs. ACh. Scale bar represents $200 \mu \mathrm{m}$.

0.01) and such protective effect of ACh was abolished by atropine, indicating that MAChR is crucially involved in the ACh-mediated suppression of ROS (Fig. 2). In addition, ACh or atropine treatment did not affect ROS level of control cells.

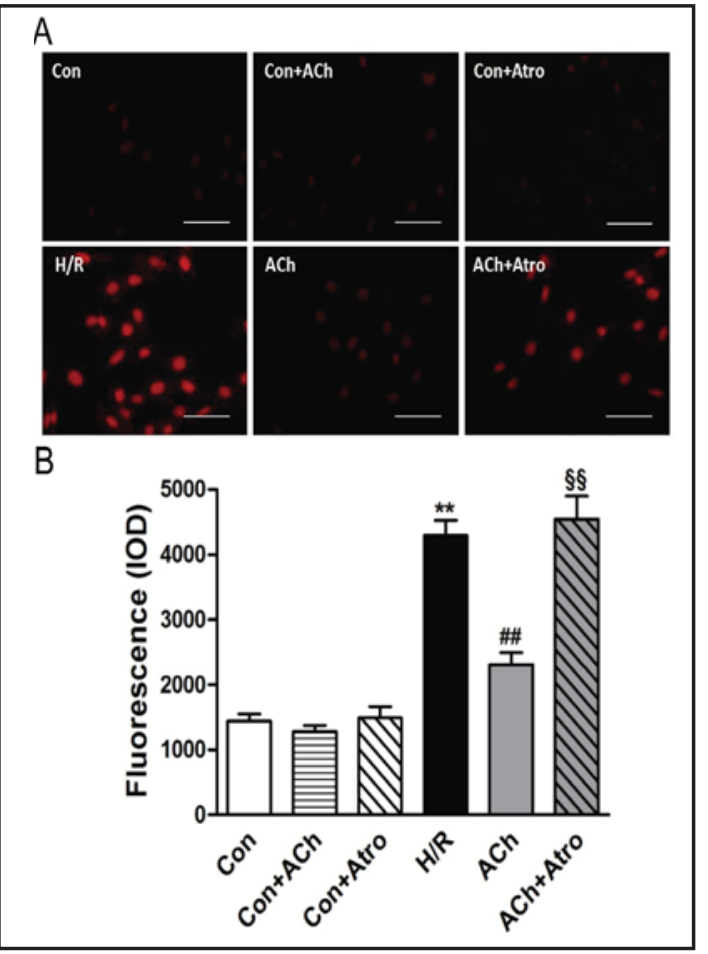




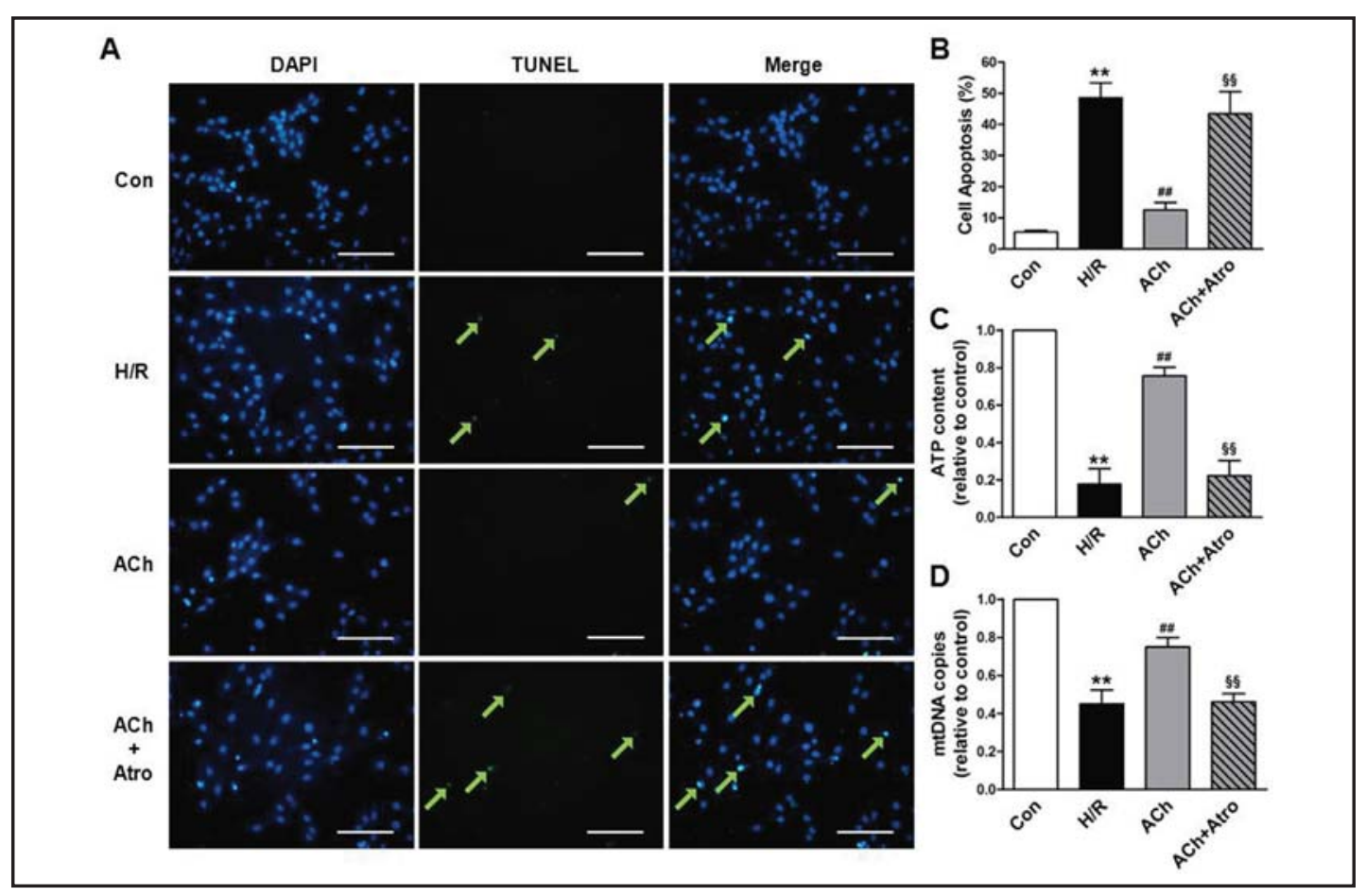

Fig. 3. ACh attenuated cell apoptosis and mitochondrial dysfunction induced by H/R injury. A-B: Representative fluorescent microscope pictures and apoptosis ratio histogram of TUNEL assay in different group. C: ACh enhanced cellular ATP level. D: ACh increased quantity of mitochondrial DNA by Real-time PCR assay. 18S rRNA was employed as an internal control. Con: Cells were kept in normal condition for corresponding time; H/R: Cells were subjected to $8 \mathrm{hrs}$ of hypoxia following $2 \mathrm{hrs}$ of reoxygenation; ACh: Cells were treated with $10^{-5} \mathrm{M}$ ACh during reoxygenation; ACh+Atro: Cells were treated with $10^{-5} \mathrm{M}$ ACh and $10^{-4} \mathrm{M}$ atropine during reoxygenation. ${ }^{* *} P<0.01$ vs. Con; ${ }^{\# \#} P<0.01$ vs. H/R; $\$ \$ P<0.01$ vs. ACh. Scale bar represents $500 \mu \mathrm{m}$.

\section{ACh attenuated cell apoptosis and mitochondrial dysfunction induced by H/R injury}

As shown in Fig. 3A and B, H/R-induced increase in TUNEL-positive nuclei in H9c2 cells were inhibited by ACh $(P<0.01)$. Treatment of cells with ACh plus atropine abolished the anti-apoptosis effects of ACh. We also detected the effects of ACh on ATP synthesis and mtDNA copy. H/R injury significantly depleted cellular ATP content in H9c2 cells. ACh treatment largely increased cellular ATP production, apparently favorable to the cell survival $(P<0.01$, Fig. 3C). In addition, mtDNA copy, which directly reflects mitochondrial function, was quantified by real-time PCR. As shown in Fig. 3D, ACh significantly reversed I/R-induced reduction in mtDNA replication $(P<0.01)$. Atropine abolished the rejuvenation effect of ACh on the H/R-induced mtDNA impairment. Together these results indicate that ACh decrease ROS production through MAChR, and thereby decrease cellular apoptosis and improve mitochondrial function, thus protecting cardiomyocytes against I/R injury. In addition, ACh or atropine treatment did not affect apoptosis, ATP synthesis and mtDNA copy of control cells (data not shown).

\section{ACh enhanced the protein expressions and activities of SOD1 and SOD2}

SOD has been emerged as a critical ROS-detoxifying enzyme. Therefore, in the present study we examined the changes of SOD following ACh treatment. A marked decrease in the SOD1 and SOD2 protein levels was observed following H/R injury $(P<0.01)$. ACh significantly reversed H/R-induced reduction in the expressions of SOD1 and SOD2, while atropine abolished these effects (Fig. 4A and B). Determination of activities of SOD1 and SOD2 with Assay Kit revealed similar results (Fig. 4C and D). 


\section{Cellular Physiology $\quad$ Cell Physiol Biochem 2014;34:1614-1625 and Biochemistry \begin{tabular}{l|l} 
DOI: 10.1159/000366364 \\
Published onIne: November 03, 2014 & $\begin{array}{l}\text { O 2014 S. Karger AG, Basel } \\
\text { www.karger.com/cpb }\end{array}$ \\
\cline { 2 - 3 }
\end{tabular}

Fig. 4. ACh enhanced expressions and activities of SOD1 and SOD2. A, B: ACh increased the protein expressions of SOD1 and SOD2. C, $\mathrm{D}$ : ACh increased the activities of SOD1 and SOD2. Con: Cells were kept in normal condition for corresponding time; H/R: Cells were treated for 2 hrs of reoxygenation after $8 \mathrm{hrs}$ of hypoxia; ACh: Cells were treated with $10^{-5} \mathrm{M}$ ACh during reoxygenation; ACh+Atro: Cells were

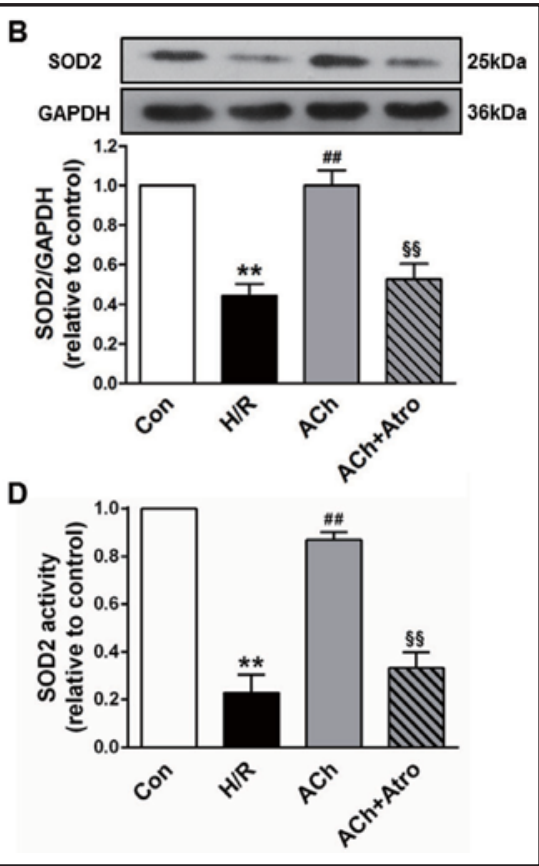

treated with $10^{-5} \mathrm{M}$ ACh and $10^{-4} \mathrm{M}$ atropine during reoxygenation. ${ }^{* *} P<0.01$ vs. Con; ${ }^{\# \#} P<0.01$ vs. H/R; $\S \S P<0.01$ vs. ACh.

Fig. 5. ACh-mediated antioxidant effect requires transcriptional factors PGC-1 $\alpha$. (A). ACh increased the protein expression of PGC- $1 \alpha$. (B). PGC- $1 \alpha$ protein expression after PGC-1 $\alpha$ siRNA transfection. (C-D). PGC- $1 \alpha$ siRNA increases the superoxide in the treatment of ACh during reoxygenation. (E). SOD1 protein expression after PGC-1 $\alpha$ siRNA transfection. (F). SOD2 protein expression after PGC- $1 \alpha$ siRNA transfection. Con: Cells were kept in normal condition for corresponding time; H/R: Cells were treated for $2 \mathrm{hrs}$ of reoxygenation after $8 \mathrm{hrs}$ of hypoxia; ACh: Cells were treated with $10^{-5} \mathrm{M} \mathrm{ACh} \mathrm{du-}$ ring reoxygenation; $\mathrm{ACh}+$ Atro: Cells were treated with $10^{-5} \mathrm{M}$ $\mathrm{ACh}$ and $10^{-4} \mathrm{M}$ atropine during reoxygenation. ns: no significant; ${ }^{* *} P<0.01$ vs. Con; ${ }^{\# \#} P<0.01$ vs. H/R; ${ }^{\S} P<0.01 v s$. ACh. Scale bar represents $200 \mu \mathrm{m}$.

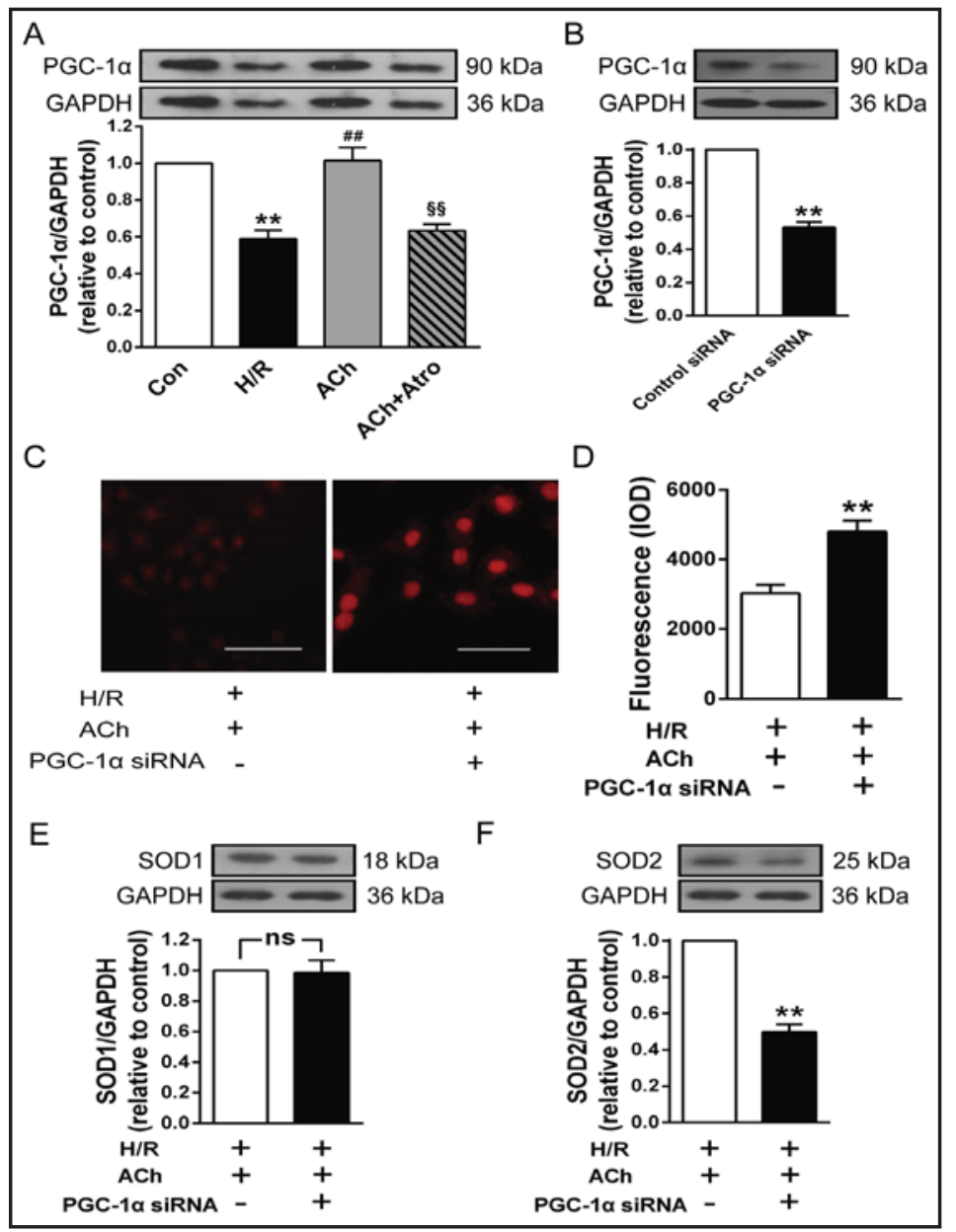




\section{Cellular Physiology Cell Physiol Biochem 2014;34:1614-1625

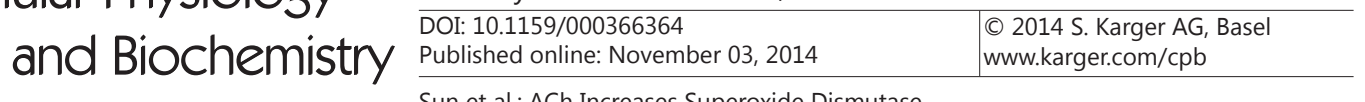

ACh-mediated antioxidant effect requires transcriptional factors $P G C-1 \alpha$

PGC- $1 \alpha$ has been reported to participate in the regulation of ROS-detoxifying enzymes. Thus, we detected the activation of PGC- $1 \alpha$. As shown in Fig. 5A, H/R decreased PGC- $1 \alpha$ expression, which was restored by ACh treatment. And this effect of ACh was inhibited by atropine. To further examine whether ACh induces SOD expression through regulation of PGC- $1 \alpha$, RNA interference was performed to knockdown PGC- $1 \alpha$. PGC- $1 \alpha$ siRNA reduced the levels of PGC-1 $\alpha$ expression by $47 \%(P<0.01$, Fig. 5B). Compared with the NC siRNA group, cells transfected with PGC-1 $\alpha$ siRNA displayed higher superoxide fluorescence intensity (Fig. 5C and D). Meanwhile, in the absence of PGC-1 $\alpha$, the expression of SOD2 is drastically decreased $(P<0.01)$. While PGC-1 $\alpha$ siRNA did not significantly affect SOD1 protein level compared with the NC siRNA group (Fig. 5E and F). These data suggest PGC- $1 \alpha$ is necessary for SOD2 regulation and ACh works through a PGC-1 $\alpha$-independent mechanism to regulate SOD1.

\section{ACh-mediated induction of SOD2 requires $P G C-1 \alpha$ and FoxO3a interaction}

Recent advances displayed that Fox03a is a direct transcriptional regulator of PGC- $1 \alpha$ expression in human umbilical vein endothelial cells. Therefore, it would be expected to also be involved in ACh-mediated induction of SOD2 in H9c2 cells. Fig. 6A showed that the phosphorylation of FoxO3a was enhanced without changes in the total amount of FoxO3a in $\mathrm{H} / \mathrm{R}$ group. ACh strongly prevented the prominent increase in FoxO3a phosphorylation

Fig. 6. ACh-mediated antioxidant effect requires transcriptional factors Fox03a. (A). ACh decreased the phosphorylation level of FoxO3a. (B). FoxO3a protein expression after PGC- $1 \alpha$ siRNA transfection. E: FoxO3a protein expression after Fox03a siRNA transfection. (C): SOD1 protein expression after FoxO3a siRNA transfection. (D): SOD2 protein expression after Fox03a siRNA transfection. (E). PGC-1 $\alpha$ protein expression after FoxO3a siRNA transfection. (F). FoxO3a protein expression after PGC- $1 \alpha$ siRNA transfection ${ }^{* *} P<0.01$ vs. control siRNA. Con: Cells were kept in normal condition for corresponding time; H/R: Cells were treated for 2 hrs of reoxygenation after 8 hrs of hypoxia; ACh: Cells were treated with $10^{-5} \mathrm{M}$ ACh during reoxygenation; $\mathrm{ACh}+\mathrm{Atro}$ : Cells were treated with $10^{-5} \mathrm{M}$ ACh and $10^{-4} \mathrm{M}$ atropine during reoxygenation. ns: no significant; ${ }^{* *} P<0.01$ vs. Con; ${ }^{\# \#} P<0.01$ vs. H/R; ${ }^{\S} P<0.01$ vs. ACh.

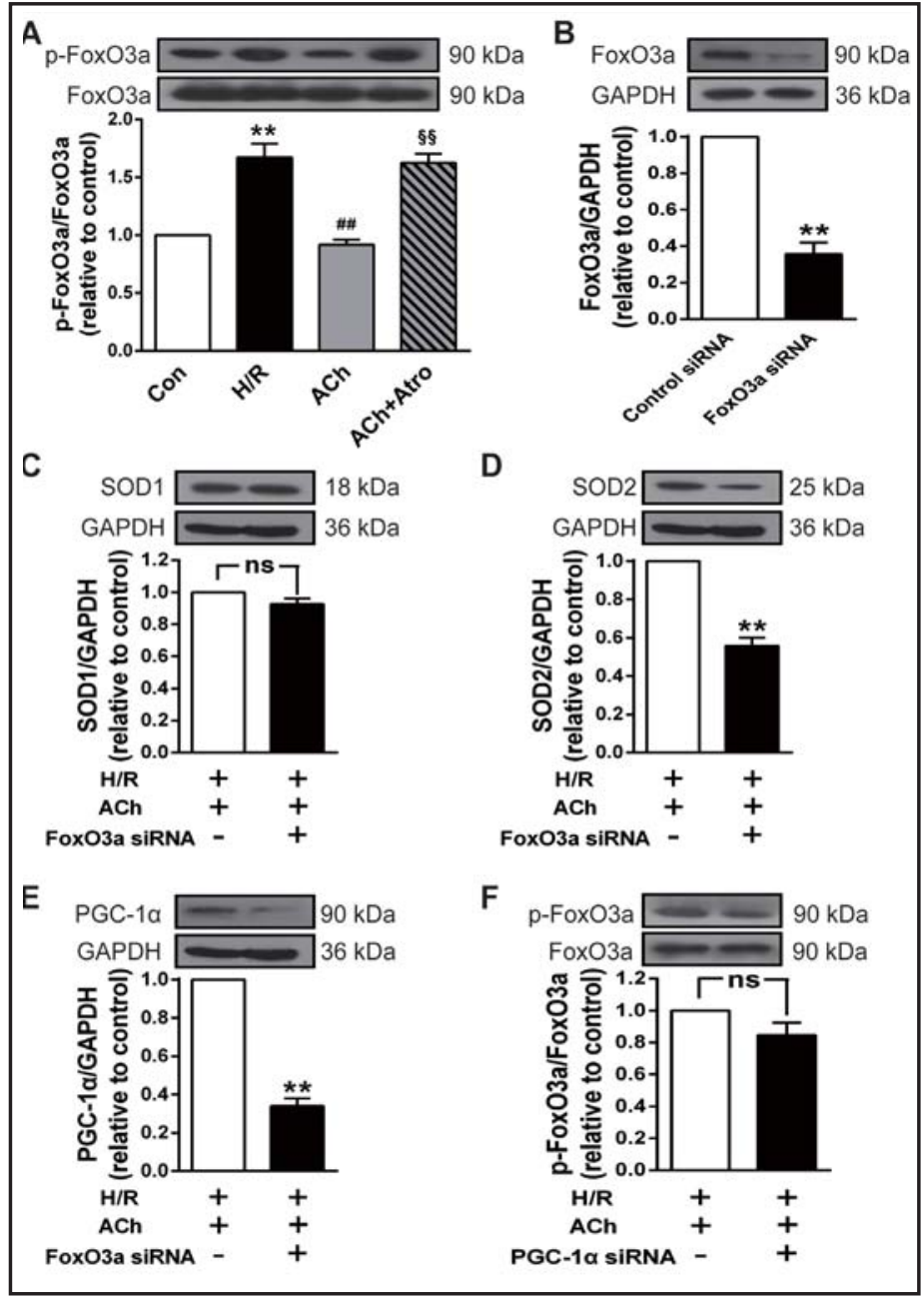




\section{Cellular Physiology and Biochemistry}

Cell Physiol Biochem 2014;34:1614-1625

DOI: 10.1159/000366364

Published onIIne: November 03, 2014

2014 Sarger AG, Basel

www.karger.com/cpb

caused by H/R injury. And this effect of ACh was inhibited by atropine. FoxO3a siRNA reduced the levels of FoxO3a expression by 81\% $(P<0.01$, Fig. 6B). Likewise, PGC- $1 \alpha$-dependent induction of SOD2 is impaired in the absence of FoxO3a $(P<0.01)$. However, FoxO3a siRNA did not significantly affect SOD1 protein level compared with the NC siRNA group (Fig. 6C and D). It is of note that knockdown of FoxO3a significantly decreased the expression of PGC$1 \alpha$, indicating that FoxO3a directly regulates the transcription of PGC- $1 \alpha$. Moreover, PGC- $1 \alpha$ siRNA had no significant effect on phosphorylation and expression of FoxO3a (Fig. 6E and F). Our results indicate that $\mathrm{ACh}$ regulates FoxO3a activation, leading to corresponding changes in PGC- $1 \alpha$ and its target genes, including those involved in ROS detoxification.

\section{Discussion}

Recent advances in understanding the cardioprotective effects of vagal regulation lead to innovative therapeutic strategies for treating myocardial reperfusion-associated oxidative stress and organ dysfunction. The present study provides the first evidence that ACh activates SOD2 within mitochondria through FoxO3a/PGC- $1 \alpha$ signaling pathway and up-regulates SOD1 in the cytoplasm, thus protecting against oxidative injury and cellular apoptosis induced by H/R (Fig. 7). Our findings further elucidate the mechanism of ROS elimination by vagal nerve regulation, which may play an important role in the modulation of the myocardial redox state.

An abnormal production of ROS has been proposed to be one important mechanism of I/R injury. Superoxide is a precursor of the other types of ROS, such as hydrogen peroxide and hydroxyl radicals. The content of superoxide can directly reflect the overall level of ROS. All known organisms must have the form of superoxide scavenging activity clearly indicates the importance of eliminating superoxide. The antioxidant enzyme SOD is the only enzyme capable of detoxifying superoxide in aerobic organisms. SOD1 is present in the cytosol and SOD2 is primarily located in mitochondria. Thus, SOD level greatly contributed to the cellular anti-oxidative activity [5]. Our results have indicated that ACh have a protective role in resistance to $\mathrm{H} / \mathrm{R}$-induced oxidative stress through the regulation of antioxidant gene SOD. Such a finding is in agreement with our recent study, showing that VNS enhanced the expressions of SOD1 and SOD2 in rat myocardium after myocardial infarction [28]. In addition to SOD, other antioxidants like catalase and glutathione reductase, also play important roles in ROS scavenging. It remains to be seen whether VNS or ACh exerts beneficial effects on other antioxidants.

Fig. 7. Proposed schematic illustration of the mechanism by which ACh exerts cardioprotection against H/R injury in the present study. ACh activates Fox03a and PGC-1 $\alpha$ via MAChR. Then these two transcriptional factors co-promote transcription of SOD2 and lead to decreased superoxide anion within mitochondria. On the other hand, ACh enhanced SOD1 expression, which could decrease superoxide anion dispersed throughout the cytoplasm. Such ROS eliminating effect protects cells and mitochondria against injury induced by $H / R$. ACh, acetylcholine; MAChR, muscarinic acetylcholine receptor; FoxO3a, forkhead box subfamily 03a; PGC-1 $\alpha$, peroxisome proliferator activated receptor co-activator $1 \alpha$; SOD1, copper/zinc superoxide dismutase; SOD2, manganese superoxide dismutase; ROS, reactive oxygen species.

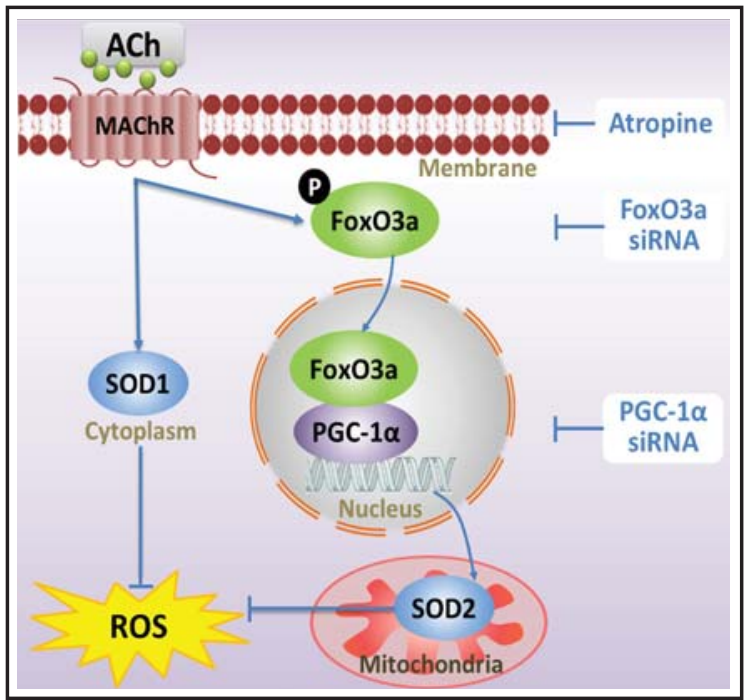




\section{Cellular Physiology and Biochemistry}

Cell Physiol Biochem 2014;34:1614-1625

\begin{tabular}{l|l}
\hline DOI: $10.1159 / 000366364$ & (C) 2014 S. Karger AG, Basel
\end{tabular}

Sun et al.: ACh Increases Superoxide Dismutase

Mitochondria are thought to be a major source of ROS as well as a major target for ROS damage [29]. Based on data from previous studies, it is plausible to speculate that ACh decreased mitochondrial ROS through up-regulating SOD2, thus protecting mitochondria against oxidative injury. The favorable effect on mitochondria further contributes to the cardioprotection. A newly report confirmed that ACh inhibited mitochondrial quantity deficiency and morphological abnormality through PGC- $1 \alpha$-associated regulatory program [30]. Together these findings support the concept that mitochondrial regulation acts as a mechanism for ACh-induced beneficial effects, and decreased mitochondrial oxidative damage contributes to the mitochondrial protection conferred by ACh. In addition, atropine (muscarinic receptor antagonist) abolished the rejuvenation effects of ACh on cardiomyocytes, indicating that MAChR is crucially involved in the cholinergic control of the heart. So far, five muscarinic receptors subtype, including $M_{1}, M_{2}, M_{3}, M_{4}$ and $M_{5}$, have been expressed and cloned in mammalian hearts. Pharmacologic evidence demonstrates that most functional responses are related to activation of $\mathrm{M}_{2} \mathrm{AChR}$ in the heart [31]. Our previous studies have suggested an important role for $\mathrm{M}_{2} \mathrm{AChR}$ in the ACh-induced protection in cardiomyocytes $[24,26]$. Therefore, $\mathrm{M}_{2} \mathrm{AChR}$ might be also involved in the ACh-mediated ROS scavenging in the present experiment. Further studies are clearly required to elucidate this issue.

Our data has indicated that ACh enhanced the protein expressions and activities of SOD1 and SOD2. However, knockdown of FoxO3a or PGC-1 $\alpha$ by siRNA did not significantly affect SOD1 expression. It is generally accepted that Fox03a proteins are expressed in developing and adult cardiomyocytes and regulate diverse cellular functions, including proliferation, metabolism and apoptosis in mitochondria. PGC- $1 \alpha$ has been identified as a master regulator of energy metabolism and mitochondrial biogenesis. Numerous studies have demonstrated that these two transcription factors are directly involved in the regulation of SOD2. However, there is no evidence to support direct regulatory effect of these factors on SOD1. This may be explained by the fact that SOD1 is present in the cytoplasm and these two transcription factors targets are mainly located in the mitochondria. Recently, it has been suggested that nuclear factor erythroid-2-related factor 2 (Nrf2) is an emerging regulator of cellular resistance to oxidants and regulates antioxidant enzymes including SOD1 [32]. It will thus be interesting to see whether ACh activates SOD1 through regulation of Nrf2 in the future studies.

Olmos et al. have suggested that Fox03a and PGC-1 $\alpha$ interact directly in the vascular endothelial cells and locate on the same promoter regions to regulate antioxidant genes including SOD2. In addition, FoxO3a directly regulate the transcription of PGC-1 $\alpha[16,33]$. In our study, FoxO3a siRNA suppressed PGC- $1 \alpha$ expression, while PGC- $1 \alpha$ siRNA did not have a significant effect on Fox03a expression. Taken together, our results allow us to propose that, ACh inhibits phosphorylation of FoxO3a, and subsequently activated FoxO3a induces PGC- $1 \alpha$ expression and interact with PGC- $1 \alpha$ to regulate the expression and activity of SOD2, which could decrease ROS within mitochondria. The present study focused on ACh-mediated ROS detoxification and related signal pathway in the cellular model, providing molecular targets against oxidative stress and evidence for drug therapy. Further studies examining the antioxidative effect of ACh or cholinergic drugs in in vivo experimental animals are warranted.

In conclusion, our data suggest that ACh ameliorates H/R injury in H9c2 cells through the action of SODs both in the mitochondria and cytosol, and thereby promoting ROSdetoxifying capacity. The protective effects of ACh against oxidative injury appear to be multifactorial through inhibition of ROS production and promoting ROS elimination, which may help to identify potential novel therapeutic targets of vagal nerve and develop future cardioprotective strategies for myocardial I/R injury.

\section{Acknowledgements}

This work is supported by the Major International (Regional) Joint Research Project of National Natural Science Foundation of China (No. 81120108002), National Natural Science 


\section{Cellular Physiology and Biochemistry}

Cell Physiol Biochem 2014;34:1614-1625

\begin{tabular}{l|l}
\hline DOI: $10.1159 / 000366364$ & (C) 2014 S. Karger AG, Basel
\end{tabular}

Sun et al.: ACh Increases Superoxide Dismutase

Foundation of China (Key Program, No. 30930105; General Program, No. 81170176), CMB Distinguished Professorships Award (No. F510000/G16916404), Specialized Research Fund for the Doctoral Program of Higher Education (No. 20130201130008) and Natural Science Foundation of Shaanxi Province (No. 2012JZ4001).

\section{References}

$\checkmark 1$ Ibanez B, Fuster V, Jimenez-Borreguero J, Badimon JJ: Lethal myocardial reperfusion injury: A necessary evil? Int J Cardiol 2011;151:3-11.

2 Prasad A, Stone GW, Holmes DR, Gersh B: Reperfusion injury, microvascular dysfunction, and cardioprotection: The "dark side" of reperfusion. Circulation 2009;120:2105-2112.

-3 Murphy E, Steenbergen C: Mechanisms underlying acute protection from cardiac ischemia-reperfusion injury. Physiol Rev 2008;88:581-609.

4 Munzel T, Gori T, Bruno RM, Taddei S: Is oxidative stress a therapeutic target in cardiovascular disease? Eur Heart J 2010;31:2741-2748.

-5 Van Raamsdonk JM, Hekimi S: Superoxide dismutase is dispensable for normal animal lifespan. Proc Natl Acad Sci U S A 2012;109:5785-5790.

6 Limon-Pacheco J, Gonsebatt ME: The role of antioxidants and antioxidant-related enzymes in protective responses to environmentally induced oxidative stress. Mutat Res 2009;674:137-147.

-7 Afanas'Ev I: ROS and RNS signaling in heart disorders: Could antioxidant treatment be successful? Oxid Med Cell Longev 2011;2011:293769.

-8 Hamanaka RB, Chandel NS: Mitochondrial reactive oxygen species regulate cellular signaling and dictate biological outcomes. Trends Biochem Sci 2010;35:505-513.

-9 Baines CP: The mitochondrial permeability transition pore and ischemia-reperfusion injury. Basic Res Cardiol 2009;104:181-188.

10 Patten IS, Arany Z: PGC-1 coactivators in the cardiovascular system. Trends Endocrinol Metab 2012;23:9097.

11 Kong X, Wang R, Xue Y, Liu X, Zhang H, Chen Y, Fang F, Chang Y: Sirtuin 3, a new target of PGC-1alpha, plays an important role in the suppression of ROS and mitochondrial biogenesis. PLoS One 2010;5:e11707.

12 Lu Z, Xu X, Hu X, Fassett J, Zhu G, Tao Y, Li J, Huang Y, Zhang P, Zhao B, Chen Y: PGC-1 alpha regulates expression of myocardial mitochondrial antioxidants and myocardial oxidative stress after chronic systolic overload. Antioxid Redox Signal 2010;13:1011-1022.

13 Sengupta A, Molkentin JD, Paik JH, DePinho RA, Yutzey KE: FoxO transcription factors promote cardiomyocyte survival upon induction of oxidative stress. J Biol Chem 2011;286:7468-7478.

14 Maiese K, Chong ZZ, Hou J, Shang YC: Oxidative stress: Biomarkers and novel therapeutic pathways. Exp Gerontol 2010;45:217-234.

15 Ronnebaum SM, Patterson C: The FoxO family in cardiac function and dysfunction. Annu Rev Physiol 2010;72:81-94.

- 16 Olmos Y, Valle I, Borniquel S, Tierrez A, Soria E, Lamas S, Monsalve M: Mutual dependence of Foxo3a and PGC-1alpha in the induction of oxidative stress genes. J Biol Chem 2009;284:14476-14484.

17 Kobayashi M, Massiello A, Karimov JH, Van Wagoner DR, Fukamachi K: Cardiac autonomic nerve stimulation in the treatment of heart failure. Ann Thorac Surg 2013;96:339-345.

18 Zhao M, Sun L, Liu JJ, Wang H, Miao Y, Zang WJ: Vagal nerve modulation: A promising new therapeutic approach for cardiovascular diseases. Clin Exp Pharmacol Physiol 2012;39:701-705.

19 Su DF: Parasympathetic nervous system: A new therapeutic target in cardiovascular disease? Clin Exp Pharmacol Physiol 2011;38:290-291.

20 Hamann JJ, Ruble SB, Stolen C, Wang M, Gupta RC, Rastogi S, Sabbah HN: Vagus nerve stimulation improves left ventricular function in a canine model of chronic heart failure. Eur J Heart Fail 2013; 15:1319-1326

21 Shinlapawittayatorn K, Chinda K, Palee S, Surinkaew S, Thunsiri K, Weerateerangkul P, Chattipakorn S, KenKnight BH, Chattipakorn N: Low-amplitude, left vagus nerve stimulation significantly attenuates ventricular dysfunction and infarct size through prevention of mitochondrial dysfunction during acute ischemia-reperfusion injury. Heart Rhythm 2013;10:1700-1707. 


\section{Cellular Physiology and Biochemistry}

Cell Physiol Biochem 2014;34:1614-1625

Sun et al.: ACh Increases Superoxide Dismutase

-22 De Ferrari GM, Crijns HJ, Borggrefe M, Milasinovic G, Smid J, Zabel M, Gavazzi A, Sanzo A, Dennert R, Kuschyk J, Raspopovic S, Klein H, Swedberg K, Schwartz PJ: Chronic vagus nerve stimulation: A new and promising therapeutic approach for chronic heart failure. Eur Heart J 2011;32:847-855.

23 Tsutsumi T, Ide T, Yamato M, Kudou W, Andou M, Hirooka Y, Utsumi H, Tsutsui H, Sunagawa K: Modulation of the myocardial redox state by vagal nerve stimulation after experimental myocardial infarction. Cardiovasc Res 2008;77:713-721.

24 Li DL, Liu JJ, Liu BH, Hu H, Sun L, Miao Y, Xu HF, Yu XJ, Ma X, Ren J, Zang WJ: Acetylcholine inhibits hypoxia-induced tumor necrosis factor-alpha production via regulation of MAPKs phosphorylation in cardiomyocytes. J Cell Physiol 2011;226:1052-1059.

25 Liu JJ, Li DL, Zhou J, Sun L, Zhao M, Kong SS, Wang YH, Yu XJ, Zhou J, Zang WJ: Acetylcholine prevents angiotensin II-induced oxidative stress and apoptosis in H9c2 cells. Apoptosis 2011;16:94-103.

26 Miao Y, Zhou J, Zhao M, Liu J, Sun L, Yu X, He X, Pan X, Zang W: Acetylcholine attenuates hypoxia/ reoxygenation-induced mitochondrial and cytosolic ROS formation in H9c2 cells via M2 acetylcholine receptor. Cell Physiol Biochem 2013;31:189-198.

27 Watkins SJ, Borthwick GM, Arthur HM: The H9C2 cell line and primary neonatal cardiomyocyte cells show similar hypertrophic responses in vitro. In Vitro Cell Dev Biol Anim 2011;47:125-131.

28 Kong SS, Liu JJ, Yu XJ, Lu Y, Zang WJ: Protection against Ischemia-Induced oxidative stress conferred by vagal stimulation in the rat heart: Involvement of the AMPK-PKC pathway. Int J Mol Sci 2012;13:1431114325.

29 Pagliaro P, Moro F, Tullio F, Perrelli MG, Penna C: Cardioprotective pathways during reperfusion: Focus on redox signaling and other modalities of cell signaling. Antioxid Redox Signal 2011;14:833-850.

-30 Sun L, Zhao M, Yu XJ, Wang H, He X, Liu JK, Zang WJ: Cardioprotection by acetylcholine: A novel mechanism via mitochondrial biogenesis and function involving the PGC-1alpha pathway. J Cell Physiol 2013;228:1238-1248.

-31 Eglen RM: Overview of muscarinic receptor subtypes. Handb Exp Pharmacol 2012;208:3-28.

-32 Ma Q: Role of nrf2 in oxidative stress and toxicity. Annu Rev Pharmacol Toxicol 2013;53:401-426.

-33 Olmos Y, Sanchez-Gomez FJ, Wild B, Garcia-Quintans N, Cabezudo S, Lamas S, Monsalve M: SirT1 regulation of antioxidant genes is dependent on the formation of a Fox03a/PGC-1alpha complex. Antioxid Redox Signal 2013;19:1507-1521. 
In the article by Sun et al., entitled "Acetylcholine Promotes ROS Detoxification Against Hypoxia/reoxygenation-Induced Oxidative Stress Through FoxO3a/PGC-1alpha Dependent Superoxide Dismutase" [Cell Physiol Biochem 2014;34:1614-1625 (DOI: $10.1159 / 000366364)]$, there is an error in Figure 5A. The correct figure with legend is reproduced here. The authors sincerely apologize for this error.

Fig. 5. ACh-mediated antioxidant effect requires transcriptional factors PGC- $1 \alpha$. (A). ACh increased the protein expression of PGC- $1 \alpha$. (B). PGC- $1 \alpha$ protein expression after PGC- $1 \alpha$ siRNA transfection. (C-D). PGC- $1 \alpha$ siRNA increases the superoxide in the treatment of ACh during reoxygenation. (E). SOD1 protein expression after PGC- $1 \alpha$ siRNA transfection. (F). SOD2 protein expression after PGC- $1 \alpha$ siRNA transfection. Con: Cells were kept in normal condition for corresponding time; H/R: Cells were treated for 2 hrs of reoxygenation after 8 hrs of hypoxia; ACh: Cells were treated with $10^{-5} \mathrm{M}$ ACh during reoxygenation; $\mathrm{ACh}+$ Atro: Cells were treated with $10^{-5} \mathrm{M} \mathrm{ACh}$ and $10^{-4} \mathrm{M}$ atropine during reoxygenation. ns: no significant; ${ }^{* *} P<$ 0.01 vs. Con; ${ }^{\# \#} P<0.01$ vs. H/R; $\S \S P<0.01$ vs. ACh.

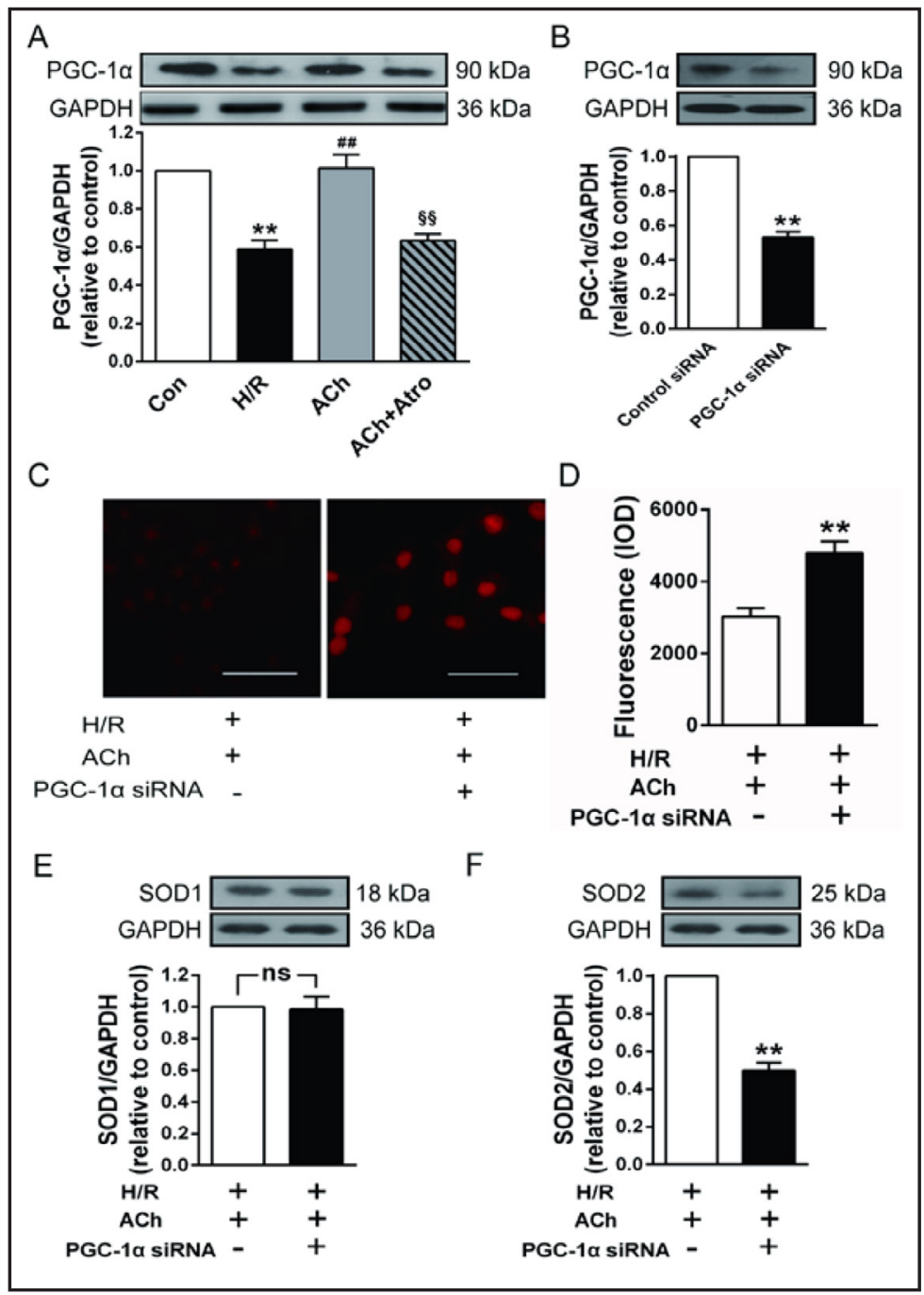

